\title{
ON MEAN VALUES \\ OF RANDOM MULTIPLICATIVE FUNCTIONS
}

\author{
YUK-KAM LAU, GÉRALD TENENBAUM, AND JIE WU
}

(Communicated by Richard C. Bradley)

\begin{abstract}
Let $\mathscr{P}$ denote the set of primes and $\{f(p)\}_{p \in \mathscr{P}}$ be a sequence of independent Bernoulli random variables taking values \pm 1 with probability $1 / 2$. Extending $f$ by multiplicativity to a random multiplicative function $f$ supported on the set of squarefree integers, we prove that, for any $\varepsilon>0$, the estimate $\sum_{n \leqslant x} f(n) \ll \sqrt{x}(\log \log x)^{3 / 2+\varepsilon}$ holds almost surely, thus qualitatively matching the law of the iterated logarithm, valid for independent variables. This improves on corresponding results by Wintner, Erdös and Halász.
\end{abstract}

\section{INTRODUCTION}

In many problems of an arithmetic nature, probabilistic models serve as heuristic support, sometimes leading to plain solutions. For instance, the link between the distribution of zeros of the Riemann $\zeta$-function and random matrix theory has been extensively studied in recent years; see in particular Montgomery's pioneering article [11, and Katz and Sarnak's important monograph 9] for a more general theory.

It is well known that equivalent forms of the Riemann hypothesis $(\mathrm{RH})$ may be stated in terms of mean values of multiplicative functions. The latest result in this direction, due to Soundararajan [15], states that, if $\mu$ designates the Möbius function, RH holds if, and only if, we have

$$
\sum_{n \leqslant x} \mu(n) \ll_{\varepsilon} \sqrt{x} \mathrm{e}^{(\log x)^{1 / 2+\varepsilon}} \quad(x \geqslant 3),
$$

for all $\varepsilon>0$. The best known estimate to date in this direction is the KorobovVinogradov bound

$$
\sum_{n \leqslant x} \mu(n) \ll x \mathrm{e}^{-c_{1}(\log x)^{3 / 5}\left(\log _{2} x\right)^{-1 / 5}} \quad(x \geqslant 3),
$$

where $c_{1}$ is a positive constant. Here and in the sequel, we let $\log _{k}$ denote the $k$-fold iterated logarithm.

A probabilistic approach to this question is therefore of great interest. It has been stated by many authors that $\mathrm{RH}$ is almost always true. However such a statement heavily depends on the nature of the random model that is chosen to

Received by the editors December 4, 2010 and, in revised form, December 5, 2010 and June 30, 2011

2010 Mathematics Subject Classification. Primary 11N37; Secondary 11K99, 60F15.

Key words and phrases. Random multiplicative functions, law of iterated logarithm, Riemann hypothesis, Möbius function, mean values of multiplicative functions, Rademacher functions. 
represent the Möbius function. If one selects random independent signs $\varepsilon_{n}$, then the desired bound follows from a well-known theorem of Khintchine and Kolmogorov according to which a series $\sum_{n \geqslant 1} \varepsilon_{n} / n^{\sigma}$ is almost always convergent if, and only if, $\sigma>1 / 2$. A more precise, and actually optimal, quantitative form is given by the law of the iterated logarithm which provides the exact maximal order for $\left|\sum_{n \leqslant x} \varepsilon_{n}\right|$ i.e. $\sqrt{\{2+o(1)\} x \log _{2} x}$; see for instance [14, p. 397 .

However, as observed by Lévy [10, such a model provides only limited hints from an arithmetical viewpoint since $\varepsilon_{n}$ does not depend on $n$ in a multiplicative manner. This led Wintner [17 to consider a setting that avoids Lévy's objection, thus laying the foundation for random multiplicative function theory.

Let $(\Omega, \mathscr{T}, \mathbb{P})$ be a probability space, let $\mathscr{P}$ denote the set of primes, and let $\{f(p)\}_{p \in \mathscr{P}}$ be a sequence of independent Bernoulli random variables on $\Omega$ taking \pm 1 both with probability $1 / 2$. For each positive integer $n$, we may define a random variable $f(n)$ on $\Omega$ by

$$
f(n):=\mu(n)^{2} \prod_{p \mid n} f(p) .
$$

Clearly, $n \mapsto f(n)$ is multiplicative, so $f(n)$ is a random multiplicative function. Of course, the probability that $f=\mu$ is zero, but it may be noticed that

$$
d_{n}:=\prod_{p \mid n} p^{(1-f(p)) / 2}
$$

is a random squarefree divisor of $n$ assuming each possible value with uniform probability $1 / 2^{\omega(n)}$, where $\omega(n)$ denotes the total number of distinct prime factors of $n$, and that

$$
f(n)=\mu(n)^{2} \mu\left(d_{n}\right) \quad(n \geqslant 1) .
$$

The quantity

$$
M_{f}(x):=\sum_{n \leqslant x} f(n)
$$

thus measures the number of cancellations arising from the multiplicative structure of the random function $f$. As a heuristic support for (1.1), Wintner [17] obtained the upper bound $M_{f}(x) \ll x^{1 / 2+\varepsilon}$ for any $\varepsilon>0$ almost surely and showed that $M_{f}(x) \ll x^{1 / 2-\varepsilon}$ is false almost surely, this latter property being shared by the Möbius function. He also noted that "the chasm between [the upper and lower bound] could perhaps be bridged by an arithmetical counterpart of Khintchine's law of the iterated logarithm".

Erdős (unpublished, see [5]) investigated in greater detail how the numbertheoretic dependence among the $f(n)$ affects the magnitude of $M_{f}(x)$. He showed that the factors $x^{\varepsilon}$ and $x^{-\varepsilon}$ may be replaced, still almost surely, by $(\log x)^{c_{2}}$ and $(\log x)^{-c_{3}}$ for some positive constants $c_{2}$ and $c_{3}$.

Halász [7] made an important step forward by proving that, for suitable positive constants $c_{4}, c_{5}$, we have almost surely

$$
M_{f}(x) \ll \sqrt{x} \mathrm{e}^{c_{4} \sqrt{\log _{2} x \log _{3} x}}
$$

while

$$
M_{f}(x) \ll \sqrt{x} \mathrm{e}^{-c_{5} \sqrt{\log _{2} x \log _{3} x}}
$$

is false almost surely. 
In a very recent paper [8], Harper improved (1.5) to the assertion that, for each $\varepsilon>0$,

$$
M_{f}(x) \ll \sqrt{x} /\left(\log _{2} x\right)^{5 / 2+\varepsilon}
$$

is almost surely false.

Of course, these estimates still fall short of any conjectural bound based on the law of the iterated logarithm, or on the belief that dependence actually reduces the expected size: see problem 26, due to Halász, in the appendix of Montgomery's monograph [12], where it is asked whether the bound $M_{f}(x) \ll \sqrt{x}$ holds almost surely.

In this paper, our aim is to investigate how close one can get to optimality for an almost sure upper bound. Improving on Halász's estimates (1.4), we show that a power of an iterated logarithm is valid, on a set of probability 1 , as an upper bound for the slowly varying factor, and hence that the multiplicative structure does not disrupt statistical cancellations in a significant way. To decide whether it actually increases the amount of cancellations remains an interesting open problem. However, this could only happen in a relatively narrow range, as shown by (1.6).

We start by setting up a slightly more general probabilistic model, in which the $f(n)$ may vanish. Let $\{f(p)\}_{p \in \mathscr{P}}$ be a sequence of independent random variables on $(\Omega, \mathscr{T}, \mathbb{P})$ such that

$$
\mathbb{P}(\{f(p)=1\})=\mathbb{P}(\{f(p)=-1\})=\frac{1}{2} \kappa_{p}, \quad \mathbb{P}(\{f(p)=0\})=1-\kappa_{p},
$$

where $\kappa_{p} \in[0,1]$ fulfils the following condition, where $c$ is a positive constant:

$$
\sum_{p \leqslant x} \kappa_{p} \log p=x+O\left(x \mathrm{e}^{-2 c \sqrt{\log x}}\right) \quad(x \geqslant 2) .
$$

We obtain a random multiplicative function $f(n)$ by (1.2). Selecting $\kappa_{p}=1$ for all primes $p$, we recover Wintner's probabilistic model. With the choice $\kappa_{p}=p /(p+1)$, we obtain the probabilistic model for a real primitive Dirichlet character, as defined by Granville and Soundararajan [6] - see also [18] — with the slight difference that our $f$ has support included in the set of squarefree integers.

Theorem 1.1. Let $\varepsilon>0$. As $x \rightarrow \infty$, we have almost surely

$$
M_{f}(x) \ll \sqrt{x}\left(\log _{2} x\right)^{3 / 2+\varepsilon} .
$$

The special case $\kappa_{p} \equiv 1$ of Theorem 1.1 provides a significant improvement over the estimate (1.4). In particular, our bound now pertains to the scale predicted by the law of the iterated logarithm. In short, our result shows that random signs behave in a comparable way whether or not a multiplicative structure is imposed.

Based on Halász's method, our upper bound is obtained by following Halász's suggestion 7 for removing the $\log _{3} x$ from (1.4). With some specific, new refinements, we show that this idea leads to a much larger gain than expected; compare Lemma 3(ii) of [7] to Lemma 3.1 below.

It is also valuable to note, as did Erdös and Halász, that, in the case $\kappa_{p}=1$, the $f(p)$ may be realized as Rademacher functions. Thus, all results in this theory find a natural interpretation in the theory of orthogonal series. 


\section{Preliminary estimates}

In the sequel of this work, we let $c_{j}(j=0,1,2, \ldots)$ denote suitable positive absolute constants.

Recall that $\omega(n)$ denotes the number of distinct prime factors of an integer $n$. For real, positive numbers $m, u, v, y, z$, we define

$$
S_{m}=S_{m}(u, v ; y, z):=\sum_{u<d \leqslant v}^{y, z} \mu(d)^{2} m^{\omega(d)},
$$

where the symbol $\sum^{y, z}$ indicates a sum restricted to integers all of whose prime factors belong to the interval $] y, z]$.

Lemma 2.1. Let $\delta \in] 0,1\left[\right.$. For $y \geqslant 3, m \geqslant 1,1<u \leqslant v\left(1-1 / y^{1-\delta}\right), y<z \leqslant y^{2}$, we have

$$
S_{m} \ll \frac{(v-u) m}{\log y} \sum_{u / z<r \leqslant v / y}^{y, z} \frac{m^{\omega(r)}}{r} .
$$

Proof. We may assume with no loss of generality that $v>y$ for $S_{m}$ otherwise vanishes. Write $d=r p$, where $p$ is the largest prime factor of $d$. We plainly have

$$
S_{m} \leqslant m \sum_{u / z<r \leqslant v / y}^{y, z} m^{\omega(r)} \sum_{u / r<p \leqslant v / r} 1 .
$$

Since $(v-u) / r \geqslant y(v-u) / v \geqslant y^{\delta}$, the Brun-Titchmarsh theorem implies that the inner sum is $\ll(v-u) /(r \log y)$.

The main aim of this section is to prove Lemma 2.3 below. For this we need to estimate moments, and so appeal to the following form of a result of Bonami 2], for which Halász [7] provided an alternate proof (Lemma 2).

Lemma 2.2. Let $f(n)$ be defined by (1.7), (1.8) and (1.2). For $m \in \mathbb{N}^{*}$ and $a_{j} \in \mathbb{C}^{\mathbb{N}^{*}}(1 \leqslant j \leqslant m)$, we have

$$
\left|\mathbb{E}\left(\prod_{1 \leqslant j \leqslant m} \sum_{n \geqslant 1} a_{j}(n) f(n)\right)\right| \leqslant\left(\prod_{1 \leqslant j \leqslant m} \sum_{n \geqslant 1}\left|a_{j}(n)\right|^{2} \kappa(n)^{2 / m}(m-1)^{\omega(n)}\right)^{1 / 2},
$$

where

$$
\kappa(n):=\mu(n)^{2} \prod_{p \mid n} \kappa_{p}
$$

Moreover, we have

$$
\mathbb{E}\left(M_{f}(x)^{2}\right) \sim c x \quad(x \rightarrow \infty)
$$

with

$$
c:=\prod_{p \in \mathscr{P}}\left(1+\kappa_{p} / p\right)(1-1 / p),
$$

and, uniformly for $v \geqslant u+1 \geqslant 2$,

$$
\mathbb{E}\left(\left\{M_{f}(v)-M_{f}(u)\right\}^{4}\right) \ll v^{2 / 3}(v-u)^{4 / 3}(\log v)^{52 / 3} .
$$


Proof. When $\kappa_{p}=1$ for all $p$, the bound (2.2) follows immediately from the induction hypothesis appearing in the proof of Lemma 2 of [7. Letting $Q$ denote the set of integral squares and using an asterisk to indicate that a summation is restricted to squarefree integers, it may be written as

$$
\left|\sum_{n_{1} \geqslant 1}^{*} \cdots \sum_{\substack{n_{m} \geqslant 1 \\ n_{1} \cdots n_{m} \in Q}}^{*} \prod_{1 \leqslant j \leqslant m} a_{j}\left(n_{j}\right)\right| \leqslant\left(\prod_{1 \leqslant j \leqslant m} \sum_{n \geqslant 1}^{*}\left|a_{j}(n)\right|^{2}(m-1)^{\omega(n)}\right)^{1 / 2} .
$$

Consider the general case. We have

$$
\mathbb{E}\left(\prod_{1 \leqslant j \leqslant m} \sum_{n \geqslant 1} a_{j}(n) f(n)\right)=\sum_{n_{1} \geqslant 1}^{*} \cdots \sum_{\substack{n_{m} \geqslant 1 \\ n_{1} \cdots n_{m} \in Q}}^{*} \prod_{\substack{1 \leqslant j \leqslant m \\ 1_{1}}} a_{j}\left(n_{j}\right) \prod_{p \mid n_{1} \cdots n_{m}} \kappa_{p} .
$$

As $0 \leqslant \kappa \leqslant 1$, we plainly have $\prod_{p \mid n_{1} \cdots n_{m}} \kappa_{p} \leqslant \prod_{1 \leqslant j \leqslant m} \kappa\left(n_{j}\right)^{1 / m}$ for all squarefree $n_{j}(1 \leqslant j \leqslant m)$, with equality if, and only if, $n_{1}=\cdots=n_{m}$. Thus, the modulus of the left-hand side of (2.7) does not exceed

$$
\leqslant \sum_{n_{1} \geqslant 1}^{*} \cdots \sum_{\substack{n_{m} \geqslant 1 \\ n_{1} \cdots n_{m} \in Q}}^{*} \prod_{\substack{1 \leqslant j \leqslant m \\ \mid}}\left|a_{j}\left(n_{j}\right)\right| \kappa\left(n_{j}\right)^{1 / m}
$$

and the bound (2.2) follows from (2.6).

Selecting $m=2$ and $a_{1}(n)=a_{2}(n)=1$ in (2.7), we get

$$
\mathbb{E}\left(M_{f}(x)^{2}\right)=\sum_{n \leqslant x} \kappa(n) .
$$

The asymptotic formula (2.4) is hence an immediate consequence of a classical theorem on multiplicative functions with values in $[0,1]$; see for instance [16], Theorem I.3.12.

Finally, since $0 \leqslant \kappa \leqslant 1$, relation (2.2) with $m=4$ and Hölder's inequality imply that

$$
\mathbb{E}\left(\left\{M_{f}(v)-M_{f}(u)\right\}^{4}\right) \leqslant\left(\sum_{u<n \leqslant v} 3^{\omega(n)}\right)^{2} \leqslant\left(\sum_{n \leqslant v} 27^{\omega(n)}\right)^{2 / 3}\left(\sum_{u<n \leqslant v} 1\right)^{4 / 3} .
$$

Therefore, the required bound (2.5) follows from the classical estimate

$$
\sum_{n \leqslant v} 27^{\omega(n)} \ll v(\log v)^{26} .
$$

Remark. As mentioned in [7, Bonami [2] proved the following variant of (2.2):

$$
\mathbb{E}\left|\sum_{n \geqslant 1} a(n) f(n)\right|^{m} \leqslant\left(\sum_{n \geqslant 1}|a(n)|^{2}(m-1)^{\omega(n)}\right)^{m / 2}
$$

in which $a \in \mathbb{C}^{\mathbb{N}^{*}}$ and $m$ may assume any real value $\geqslant 2$. We shall not need such a generalization in this work.

According to (2.4), the expected order of $M_{f}(x)$ is $\sqrt{x}$. The next lemma, essentially identical to Lemma 1 of [7] (and the proof of which we provide for mere convenience), shows that, almost surely, this quantity fluctuates moderately in appropriate short intervals; in other words, the problem of bounding $M_{f}(x)$ everywhere may be reduced to doing so at suitable test-points. 
Lemma 2.3. Let $f(n)$ be defined by (1.7), (1.8) and (1.2). For any fixed constant $A>0$, there is a suitable constant $\left.c_{6}=c_{6}(A) \in\right] 0,1[$ such that, for

$$
x_{i}:=\left\lfloor e^{i^{c_{6}}}\right\rfloor \quad(i \geqslant 1),
$$

we have almost surely

$$
\max _{x_{i-1}<x \leqslant x_{i}}\left|M_{f}(x)-M_{f}\left(x_{i-1}\right)\right| \ll_{A, f} \frac{\sqrt{x_{i}}}{\left(\log x_{i}\right)^{A}} \quad(i \geqslant 1) .
$$

Proof. Assume that

$$
\max _{x_{i-1}<x \leqslant x_{i}}\left|M_{f}(x)-M_{f}\left(x_{i-1}\right)\right|>2 \sqrt{x_{i}} /\left(\log x_{i}\right)^{A}
$$

and that the maximum is attained at some integer

$$
x=x_{i-1}+\sum_{1 \leqslant j \leqslant h} 2^{\nu_{j}},
$$

where $\left\{\nu_{j}\right\}_{j=1}^{h} \in \mathbb{N}^{h}$ and $\nu_{1}>\cdots>\nu_{h} \geqslant 0$. We split $\left[x_{i-1}, x\right]$ into a disjoint union of subintervals with limit points

$$
u_{k}=x_{i-1}+\sum_{1 \leqslant j \leqslant k} 2^{\nu_{j}} \quad(0 \leqslant k \leqslant h) .
$$

Thus, there exists a pair $\left\{u_{k}, u_{k+1}\right\}$ such that

$$
\left|M_{f}\left(u_{k+1}\right)-M_{f}\left(u_{k}\right)\right|>\sqrt{x_{i}} /\left(\log x_{i}\right)^{A+1},
$$

for the number of these subintervals does not exceed $1+\left\{\log \left(x_{i}-x_{i-1}\right)\right\} / \log 2<$ $2 \log x_{i}$. Note that

$$
u_{k}=x_{i-1}+(\ell-1) 2^{m}, \quad u_{k+1}=x_{i-1}+\ell 2^{m},
$$

with $\ell=\sum_{1 \leqslant j \leqslant k} 2^{\nu_{j}-\nu_{k}}$ and $m=\nu_{k}$.

Next, we bound the total probability of the occurrence of (2.11) when (2.12) holds for some $\ell$ and $m$; this clearly dominates the probability of (2.10).

By Markov's inequality for the fourth moment and (2.5), we may write

$$
\mathbb{P}\left(\left|M_{f}(v)-M_{f}(u)\right|>\sqrt{x_{i}} /\left(\log x_{i}\right)^{A+1}\right) \ll\left(\frac{v-u}{x_{i}}\right)^{4 / 3}\left(\log x_{i}\right)^{4 A+64 / 3} .
$$

Let $u=x_{i-1}+(\ell-1) 2^{m}$ and $v=x_{i-1}+\ell 2^{m}$, where $\ell \geqslant 1, m \geqslant 0$ and $\ell 2^{m} \leqslant x_{i}-x_{i-1}$. Then by (2.13), the probability that (2.11) holds for some $u_{k}, u_{k+1}$ of the form (2.12) is

$$
\begin{aligned}
& \ll \sum_{\ell 2^{m} \leqslant x_{i}-x_{i-1}}\left(\frac{2^{m}}{x_{i}}\right)^{4 / 3}\left(\log x_{i}\right)^{4 A+64 / 3} \\
& \ll\left(\log x_{i}\right)^{4 A+64 / 3} \sum_{2^{m} \leqslant x_{i}-x_{i-1}}\left(\frac{2^{m}}{x_{i}}\right)^{4 / 3} \frac{x_{i}-x_{i-1}}{2^{m}} \\
& \ll\left(\frac{x_{i}-x_{i-1}}{x_{i}}\right)^{4 / 3}\left(\log x_{i}\right)^{4 A+64 / 3} .
\end{aligned}
$$

Set $c_{6}:=1 /(272+48 A)$. As $\left(\log x_{i}\right)^{4 A+64 / 3} \leqslant i^{(4 A+64 / 3) c_{6}}$ and $\left(x_{i}-x_{i-1}\right) / x_{i} \ll$ $1 / i^{1-c_{6}}$, we deduce that $(\underline{2.14})$ is $\ll i^{-5 / 4}$ and hence that the same bound holds for 
the probability of the event (2.10). The proof is completed by the Borel-Cantelli lemma; see, e.g., 4, Theorem 4.2.1.

Remark. Chatterjee and Soundararajan ([3], Prop. 3.1) evaluate

$$
\mathbb{E}\left(\left\{M_{f}(v)-M_{f}(u)\right\}^{4}\right)
$$

for short intervals $] u, v]$. However, inserting this estimate into the above proof would only yield to an improvement on the value of the constant $c_{6}$, with no influence on the final exponent $3 / 2$ appearing in Theorem 1.1 .

\section{Proof of Theorem 1.1}

We first establish an average estimate improving significantly over the corresponding bound obtained by Halász; see [7, formula (2).

Lemma 3.1. Let $f(n)$ be defined by (1.7), (1.8) and (1.2) and let $\left\{x_{i}\right\}_{i \geqslant 1}$ be given by (2.9). Then, for any $\varepsilon>0$, we have almost surely

$$
\frac{1}{x_{i}-x_{i-1}} \int_{x_{i-1}}^{x_{i}} M_{f}(x) \mathrm{d} x \ll_{f, \varepsilon} \sqrt{x_{i}}\left(\log _{2} x_{i}\right)^{3 / 2+\varepsilon} \quad(i \geqslant 1) .
$$

Proof. We show that large values of the integral occur with small probability and conclude by the Borel-Cantelli lemma.

We have $x_{i}=\left\lfloor e^{i^{c_{6}}}\right\rfloor$. Given a large constant $\ell_{0}$, we put $X_{\ell}:=\mathrm{e}^{2^{\ell}}$ for $\ell \geqslant \ell_{0}$, so that $X_{\ell-1}=X_{\ell}^{1 / 2}$. We consider those $x_{i}$ lying in $\left.] X_{\ell-1}, X_{\ell}\right]$, and for $\left.\alpha \in\right] 0, \frac{1}{2}[$, write

$$
y_{0}:=\exp \left\{\frac{c_{6} 2^{\ell}}{4 \ell}\right\}, \quad y_{j}:=y_{j-1}^{\mathrm{e}^{\alpha}}=y_{0}^{\mathrm{e}^{\alpha j}} \quad(j \geqslant 1),
$$

and observe that, if $J$ is minimal under the constraint $y_{J} \geqslant X_{\ell}$, then

$$
J \leqslant 1+\frac{\log \left\{4 \ell / c_{6}\right\}}{\alpha} \ll \frac{\log \ell}{\alpha} .
$$

Employing the notation

$$
\Psi_{f}(x, y):=\sum_{\substack{n \leqslant x \\ P(n) \leqslant y}} f(n) \quad(x \geqslant 0, y \geqslant 1),
$$

we split the sum $M_{f}(x)$ according to the size of the largest prime factor $P(n)$ of the summation variable $n$. For $x \in\left[x_{i-1}, x_{i}\right]$, we thus obtain

$$
\begin{aligned}
M_{f}(x) & =\sum_{\substack{n \leqslant x \\
P(n) \leqslant y_{0}}} f(n)+\sum_{1 \leqslant j \leqslant J} \sum_{y_{j-1}<d \leqslant x_{i}} \sum_{\substack{m \leqslant x / d \\
P(m) \leqslant y_{j-1}}}^{y_{j-1}, y_{j}} f(d m) \\
& =\Psi_{f}\left(x, y_{0}\right)+\sum_{1 \leqslant j \leqslant J} \sum_{y_{j-1}<d \leqslant x_{i}}^{y_{j-1}, y_{j}} f(d) \Psi_{f}\left(x / d, y_{j-1}\right) .
\end{aligned}
$$

Setting $\delta_{i}:=x_{i}-x_{i-1}$, we express accordingly the integral to be bounded as follows:

$$
\frac{1}{\delta_{i}} \int_{x_{i-1}}^{x_{i}} M_{f}(x) \mathrm{d} x=N_{i 0}(f)+\sum_{1 \leqslant j \leqslant J} N_{i j}(f),
$$


where

$$
\begin{aligned}
N_{i 0}(f) & :=\frac{1}{\delta_{i}} \int_{x_{i-1}}^{x_{i}} \Psi_{f}\left(x, y_{0}\right) \mathrm{d} x \\
N_{i j}(f) & :=\sum_{y_{j-1}<d \leqslant x_{i}}^{y_{j-1}, y_{j}} b_{i j}(d ; f) f(d) \quad(j \geqslant 1)
\end{aligned}
$$

with

$$
b_{i j}(d ; f):=\frac{1}{\delta_{i}} \int_{x_{i-1}}^{x_{i}} \Psi_{f}\left(x / d, y_{j-1}\right) \mathrm{d} x .
$$

We first establish an upper bound for the probability of the event

$$
\mathscr{A}=\mathscr{A}_{\ell}(R):=\bigcup_{X_{\ell-1}<x_{i} \leqslant X_{\ell}}\left\{\frac{1}{\delta_{i}}\left|\int_{x_{i-1}}^{x_{i}} M_{f}(x) \mathrm{d} x\right| \geqslant 2 \sqrt{x_{i}} R\right\} .
$$

To this end, we define

$$
\begin{aligned}
& \mathscr{B}_{0}=\mathscr{B}_{0}(R ; \ell):=\bigcup_{X_{\ell-1}<x_{i} \leqslant X_{\ell}}\left\{\left|N_{i 0}(f)\right| \geqslant \sqrt{x_{i}} R\right\}, \\
& \mathscr{B}_{1}=\mathscr{B}_{1}(R ; \ell):=\bigcup_{X_{\ell-1}<x_{i} \leqslant X_{\ell}}\left\{\sum_{1 \leqslant j \leqslant J}\left|N_{i j}(f)\right| \geqslant \sqrt{x_{i}} R\right\} .
\end{aligned}
$$

Clearly $\mathscr{A} \subset \mathscr{B}_{0} \cup \mathscr{B}_{1}$, so

$$
\mathbb{P}(\mathscr{A}) \leqslant \mathbb{P}\left(\mathscr{B}_{0}\right)+\mathbb{P}\left(\mathscr{B}_{1}\right) .
$$

We first estimate $\mathscr{B}_{1}$. Following Halász, we consider the filtration $\{\mathscr{T}(y)\}_{y \geqslant 1}$, where $\mathscr{T}(y)$ denotes the $\sigma$-algebra generated by the variables $f(p)$ with $p \leqslant y$. Since $0 \leqslant \kappa \leqslant 1$, we may deduce from Lemma 2.2 that, for any integer $m \geqslant 1$, we have

$$
\mathbb{E}\left(\left|N_{i j}(f)\right|^{2 m} \mid \mathscr{T}\left(y_{j-1}\right)\right) \leqslant\left(x_{i} D_{i j}(f)\right)^{m},
$$

where

$$
D_{i j}(f):=\frac{1}{x_{i}} \sum_{y_{j-1}<d \leqslant x_{i}}^{y_{j-1}, y_{j}} b_{i j}(d ; f)^{2} \mu(d)^{2}(2 m-1)^{\omega(d)} .
$$

From the Cauchy-Schwarz inequality, we see that

$$
b_{i j}(d ; f)^{2} \leqslant \frac{1}{\delta_{i}} \int_{x_{i-1}}^{x_{i}} \Psi_{f}\left(x / d, y_{j-1}\right)^{2} \mathrm{~d} x \leqslant \frac{x_{i}}{\delta_{i}} \int_{x_{i-1} / d}^{x_{i} / d} \frac{\Psi_{f}\left(t, y_{j-1}\right)^{2}}{t} \mathrm{~d} t,
$$

where we made the change of variable $t:=x / d$ and used the inequality $d=x / t \leqslant$ $x_{i} / t$. Therefore

$$
D_{i j}(f) \leqslant D_{i j}^{*}(f):=\frac{1}{\delta_{i}} \int_{1}^{x_{i} / y_{j-1}} \sum_{x_{i-1} / t<d \leqslant x_{i} / t}^{y_{j-1}, y_{j}} \mu(d)^{2}(2 m-1)^{\omega(d)} \frac{\Psi_{f}\left(t, y_{j-1}\right)^{2}}{t} \mathrm{~d} t .
$$


Applying Lemma 2.1 to the right-hand side of (3.9), we obtain

$$
\begin{aligned}
D_{i j}^{*}(f) & \ll \frac{1}{\log y_{j-1}} \sum_{r \leqslant X_{\ell}}^{y_{j-1}, y_{j}} \frac{(2 m-1)^{\omega(r)+1}}{r} \int_{x_{i-1} / r y_{j}}^{x_{i} / r y_{j-1}} \frac{\Psi_{f}\left(t, y_{j-1}\right)^{2}}{t^{2}} \mathrm{~d} t \\
& \ll \frac{m}{\log y_{j-1}} \int_{1}^{X_{\ell} / y_{j-1}} \sum_{x_{i-1} / t y_{j}<r \leqslant x_{i} / t y_{j-1}}^{y_{j-1}, y_{j}} \frac{(2 m-1)^{\omega(r)}}{r} \frac{\Psi_{f}\left(t, y_{j-1}\right)^{2}}{t^{2}} \mathrm{~d} t .
\end{aligned}
$$

Now, we observe that, for $y<\min (z, w), m \geqslant 1$, we have

$$
\sum_{r \leqslant w}^{y, z}(2 m-1)^{\omega(r)} \ll \sum_{s \leqslant w}^{y, z}(2 m-2)^{\omega(s)} \sum_{d \leqslant w / s}^{y, w} 1 \ll \frac{w}{\log y} \prod_{y<p \leqslant z}\left(1+\frac{2 m-2}{p}\right),
$$

from which we deduce by partial summation that

$$
\sum_{x_{i-1} / t y_{j}<r \leqslant x_{i} / t y_{j-1}}^{y_{j-1}, y_{j}} \frac{(2 m-1)^{\omega(r)}}{r} \ll \alpha \mathrm{e}^{c_{6} \alpha m}
$$

provided $\alpha \gg 1 / 2^{\ell} \gg 1 / \log y_{0}$. Writing

$$
I_{j \ell}:=\int_{1}^{X_{\ell}} \frac{\Psi_{f}\left(t, y_{j-1}\right)^{2}}{t^{2}} \mathrm{~d} t
$$

we thus have

$$
D_{i j}^{*}(f)^{m} \leqslant\left(\frac{c_{8} \alpha m \mathrm{e}^{c_{7} \alpha m} I_{j \ell}}{\log y_{j-1}}\right)^{m} .
$$

Let $T \geqslant 1$ be specified later. Defining the events

$$
\mathscr{C}_{j}:=\left\{I_{j \ell} \leqslant T \log y_{j-1}\right\} \quad(1 \leqslant j \leqslant J), \quad \mathscr{C}:=\bigcap_{1 \leqslant j \leqslant J} \mathscr{C}_{j},
$$

we plainly have

$$
\mathscr{B}_{1} \subset\left(\mathscr{B}_{1} \cap \mathscr{C}\right) \cup \overline{\mathscr{C}}
$$

By Lemma 2.2 and the classical estimate ([16], Th. III.5.1)

$$
\Psi(x, y):=\sum_{\substack{n \leqslant x \\ P(n) \leqslant y}} 1 \ll x^{1-1 /(2 \log y)} \quad(x \geqslant y \geqslant 2),
$$

we have $\mathbb{E}\left(I_{j \ell}\right) \ll \log y_{j-1}$, whence

$$
\mathbb{P}\left(\overline{\mathscr{C}_{j}}\right) \ll \frac{1}{T} .
$$

Moreover, since, as first noticed by Basquin [1], $\left\{I_{j \ell}\right\}_{j=0}^{J}$ is a submartingale with respect to the filtration $\left\{\mathscr{T}\left(y_{j}\right): 1 \leqslant j \leqslant J\right\}$, we actually deduce from Doob's inequality (see, e.g., [13, Theorem II.1.7) that

$$
\mathbb{P}(\overline{\mathscr{C}}) \ll \frac{\log \ell}{T} .
$$


Indeed,

$$
\mathbb{P}\left(\sup _{2^{r}<\log y_{j-1} \leqslant 2^{r+1}} \frac{I_{j \ell}}{\log y_{j-1}}>T\right) \ll \frac{1}{T}
$$

for each integer $r$ such that $c_{6} 2^{\ell} /(4 \ell \log 2)<2^{r} \leqslant 2^{\ell}$. Since there are only $\ll \log \ell$ possible values of $r$, 3.15) follows.

Applying Hölder's inequality in the form

$$
\left(\sum_{1 \leqslant j \leqslant J}\left|N_{i j}(f)\right|\right)^{2 m} \leqslant J^{2 m-1} \sum_{1 \leqslant j \leqslant J}\left|N_{i j}(f)\right|^{2 m},
$$

we derive from (3.6), (3.8), (3.9) and (3.11) that

$$
\begin{aligned}
\mathbb{P}\left(\mathscr{B}_{1} \cap \mathscr{C}\right) & \leqslant \mathbb{P}\left(\mathscr{B}_{1} \mid \mathscr{C}\right) \leqslant \sum_{X_{\ell-1}<x_{i} \leqslant X_{\ell}} \sum_{1 \leqslant j \leqslant J} \frac{\mathbb{E}\left(\left|N_{i j}(f)\right|^{2 m} \mid \mathscr{C}_{j}\right) J^{2 m-1}}{\left(x_{i} R^{2}\right)^{m}} \\
& \ll 2^{\ell / c_{6}}\left(\frac{c_{8} T J^{2} \alpha m \mathrm{e}^{c_{7} \alpha m}}{R^{2}}\right)^{m} .
\end{aligned}
$$

Finally, we bound $\mathbb{P}\left(\mathscr{B}_{0}\right)$. Using the Cauchy-Schwarz inequality, we get, as before,

$$
\begin{aligned}
\mathbb{E}\left(N_{i 0}(f)^{2}\right) & \leqslant \frac{1}{\delta_{i}} \int_{x_{i-1}}^{x_{i}} \mathbb{E}\left(\Psi_{f}\left(x, y_{0}\right)^{2}\right) \mathrm{d} x \\
& \leqslant \frac{1}{\delta_{i}} \int_{x_{i-1}}^{x_{i}} \Psi\left(x, y_{0}\right) \mathrm{d} x \ll x_{i} \mathrm{e}^{-\left(\log X_{\ell}\right) /\left(4 \log y_{0}\right)}=x_{i} 2^{-\ell / c_{6}}
\end{aligned}
$$

since $x_{i} \geqslant X_{\ell-1}=X_{\ell}^{1 / 2}$. By Markov's inequality, we then deduce that

$$
\begin{aligned}
\mathbb{P}\left(\mathscr{B}_{0}\right) & \leqslant \sum_{X_{\ell-1}<x_{i} \leqslant X_{\ell}} \mathbb{P}\left(N_{i 0}(f) \geqslant \sqrt{x_{i}} R\right) \\
& \leqslant \sum_{X_{\ell-1}<x_{i} \leqslant X_{\ell}} \frac{\mathbb{E}\left(N_{i 0}(f)^{2}\right)}{\left(\sqrt{x_{i}} R\right)^{2}} \ll \frac{2^{-\ell / c_{6}}}{R^{2}} \sum_{X_{\ell-1}<x_{i} \leqslant X_{\ell}} 1 \ll \frac{1}{R^{2}},
\end{aligned}
$$

by our choice for $x_{i}$ and $X_{\ell}$.

Collecting our estimates (3.13), (3.15), (3.16), (3.17) and inserting back into (3.7), we get

$$
\mathbb{P}(\mathscr{A}) \ll \frac{1}{R^{2}}+2^{\ell / c_{6}}\left(\frac{c_{8} T J^{2} \alpha m \mathrm{e}^{c_{7} \alpha m}}{R^{2}}\right)^{m}+\frac{\log \ell}{T} .
$$

Selecting

$$
T:=\ell^{1+\varepsilon / 2}, \quad R:=\ell^{3 / 2+\varepsilon}, \quad \alpha:=1 / \ell, \quad m:=\ell,
$$

so that $J \ll \ell \log \ell$, we conclude that

$$
\mathbb{P}(\mathscr{A}) \ll \varepsilon \frac{\log \ell}{\ell^{1+\varepsilon / 2}} .
$$

Thus, the Borel-Cantelli lemma implies that

$$
\mathbb{P}\left(\limsup _{\ell \geqslant 1} \mathscr{A}_{\ell}(R)\right)=0 .
$$

This finishes the proof of Lemma 3.1 . 
Now we are ready to prove Theorem 1.1. From the identity

$\int_{x_{i-1}}^{x_{i}} M_{f}(t) \mathrm{d} t=\delta_{i} M_{f}(x)-\delta_{i}\left\{M_{f}(x)-M_{f}\left(x_{i-1}\right\}+\int_{x_{i-1}}^{x_{i}}\left\{M_{f}(t)-M_{f}\left(x_{i-1}\right)\right\} \mathrm{d} t\right.$,

we deduce that, for all $i \geqslant 1$ and all $\left.x \in] x_{i-1}, x_{i}\right]$, we have

$$
\left|M_{f}(x)\right| \leqslant \frac{1}{\delta_{i}}\left|\int_{x_{i-1}}^{x_{i}} M_{f}(t) \mathrm{d} t\right|+2 \max _{x_{i-1}<t \leqslant x_{i}}\left|M_{f}(t)-M_{f}\left(x_{i-1}\right)\right| .
$$

Write

$$
E_{\ell}:=\left\{\sup _{X_{\ell-1}<x \leqslant X_{\ell}} \frac{\left|M_{f}(x)\right|}{\sqrt{x}\left(\log _{2} x\right)^{3 / 2+\varepsilon}}>4\right\} .
$$

From the above upper bound, Lemma 2.3 with $A=1$, and (3.19), we have

$$
\mathbb{P}\left(E_{\ell}\right) \ll(\log \ell) / \ell^{1+\varepsilon / 2} .
$$

It hence follows from the Borel-Cantelli lemma that $\mathbb{P}\left(\lim _{\sup _{\ell \rightarrow \infty}} E_{\ell}\right)=0$, as required.

\section{REFERENCES}

[1] J. Basquin, Sommes friables de fonctions multiplicatives aléatoires, Acta Arith., to appear.

[2] A. Bonami, Étude des coefficients de Fourier des fonctions de $L^{p}(G)$, Ann. Inst. Fourier, Grenoble 20, 2 (1970), 335-402. MR.0283496 (44:727)

[3] S. Chatterjee and K. Soundararajan, Random multiplicative functions in short intervals, Int. Math. Res. Notices 2012, No. 3, 479-492.

[4] K.L. Chung, A course in probability theory, Harcourt, Brace \& World, Inc., New York/ Chicago/San Francisco/Atlanta, 1968. MR0229268 (37:4842)

[5] P. Erdős, Some unsolved problems, Magyar Tud. Akad. Mat. Kut. Int. Közl. 6 (1961), 211254. MR0177846 (31:2106)

[6] A. Granville and K. Soundararajan, The distribution of values of $L\left(1, \chi_{d}\right)$, Geom. Funct. Anal. 13 (2003), no. 5, 992-1028. MR2024414 (2005d:11129)

[7] G. Halász, On random multiplicative functions, Publ. Math. Orsay, 83-4 (1983), 74-96. MR728404(85i:11081)

[8] A.J. Harper, Bounds on the suprema of Gaussian processes, and omega results for the sum of a random multiplicative function, preprint, arXiv:1012.0210.

[9] N.M. Katz and P. Sarnak, Random matrices, Frobenius eigenvalues, and monodromy, American Mathematical Society Colloquium Publications, 45. American Mathematical Society, Providence, RI, 1999. MR,1659828 (2000b:11070)

[10] P. Lévy, Sur les séries dont les termes sont des variables eventuelles indépendantes, Studia Math. 3 (1931), 119-155.

[11] H.L. Montgomery, The pair correlation of zeros of the zeta function, in: Analytic number theory (Proc. Sympos. Pure Math., Vol. XXIV, St. Louis Univ., St. Louis, Mo., 1972), pp. 181193, Amer. Math. Soc., Providence, RI, 1973. MR0337821 (49:2590)

[12] H.L. Montgomery, Ten lectures on the interface between analytic number theory and harmonic analysis, CBMS 84, AMS, Providence, RI, 1994. MR.1297543 (96i:11002)

[13] D. Revuz and M. Yor, Continuous martingales and Brownian motion (Third ed.), Grundlehren der Mathematischen Wissenschaften 293, Springer-Verlag, Berlin, 1999. MR 1725357 (2000h:60050)

[14] A.N. Shiryaev, Probability, Second edition, Graduate Texts in Mathematics 95, SpringerVerlag, New York, 1996. MR:1368405 (97c:60003)

[15] K. Soundararajan, Partial sums of the Möbius function, J. reine angew. Math. 631 (2009), 141-152. MR2542220 (2010e:11090)

[16] G. Tenenbaum, Introduction à la théorie analytique et probabiliste des nombres, Coll. Échelles, Berlin, 2008. 
[17] A. Wintner, Random factorizations and Riemann's hypothesis, Duke Math. J. 11 (1944), 267-275. MR0010160 (5:255c)

[18] J. Wu, Note on a paper by Granville and Soundararajan, J. Number Theory 123 (2007), 329-351. MR 2300818 (2008f:11096) KONG

Department of Mathematics, The University of Hong Kong, Pokfulam Road, Hong

E-mail address: yklau@maths.hku.hk

Institut Élie Cartan Nancy, NAncy-Université, CNRS \& INRIA, 54506 VAndeuvreLÈS-NANCY, FRANCE

E-mail address: gerald.tenenbaum@iecn.u-nancy.fr

Institut Élie Cartan Nancy, Nancy-Université, CNRS \& INRIA, 54506 VandeuvreLÈS-NANCY, FRANCE

E-mail address: wujie@iecn.u-nancy.fr 